\title{
A Mechanical Model of Brownian Motion
}

\author{
D. Dürr ${ }^{\star}$, S. Goldstein ${ }^{\star \star}$, and J. L. Lebowitz ${ }^{\star \star \star}$ \\ Department of Mathematics, Rutgers University, New Brunswick, NJ 08903, USA
}

\begin{abstract}
We consider a dynamical system consisting of one large massive particle and an infinite number of light point particles. We prove that the motion of the massive particle is, in a suitable limit, described by the OrnsteinUhlenbeck process. This extends to three dimensions previous results by Holley in one dimension.
\end{abstract}

\section{Introduction}

The erratic motion of a macroscopically small but microscopically large particle (visible in a microscope), in an equilibrium fluid, e.g. pollen in a liquid or a smoke particle in air, is a well known physical phenomenon going under the name of Brownian motion [1]. The physical explanation and mathematical description of this motion were first given by Einstein: it is due to the fluctuations in the force exerted on the Brownian particle by its collisions with the fluid atoms and is described by a diffusion equation for the displacement of the Brownian particle. The ultimate mathematical idealization of this phenomenon is the OrnsteinUhlenbeck process for the position and velocity of the Brownian particle $\left(\underline{X}_{t}, \underline{V}_{t}\right)$, described by the stochastic differential equations

$$
\begin{aligned}
& d \underline{X}_{t}=\underline{V}_{t} d t \\
& d \underline{V}_{t}=-a \underline{V}_{t} d t+\sqrt{D} d \underline{W}_{t}, \quad a \geqq 0, \quad D \geqq 0, \quad \underline{W}_{t}=\text { Wiener process. }
\end{aligned}
$$

The position process $\underline{X}_{t}$ converges in an appropriate limit (e.g. $a \rightarrow \infty, a^{2} / D=$ const) to a Wiener process.

A little thought shows that in order to rigorously derive the OrnsteinUhlenbeck process for the Brownian particle from the mechanical motion of the

* On leave of the Institut für Theoretische Physik I der Universität Münster. Supported by a Nato fellowship

$\star \star$ Supported by NSF Grant, No. PHY 78-03816

$\star \star \star$ Supported by NSF Grant, Phy 78-15920 
isolated system consisting of the Brownian particle plus fluid, one must consider a "suitable" limit, in which the ratio of the mass of the Brownian particle to the mass of a fluid particle becomes infinitely large.

We present here such a mechanical model for the Ornstein-Uhlenbeck process: the motion of a heavy particle of mass $M$ in an infinite ideal gas of point particles of mass $m$ in the limit $M / m \rightarrow \infty$. This is an extension to three (actually an arbitrary number of) dimensions of the one dimensional work done by Holley [2]. Previous work on higher dimensional models $[3,4]$ considered only a non mechanical Markov process.

The physical situation is as follows. Consider an infinite ideal gas of point particles of mass $m$ with independent velocities given by a probability distribution $F$, which we assume to be absolutely continuous with respect to the Lebesgue measure, i.e. $F(d \underline{v})=f(\underline{v}) d \underline{v}$. We first consider the case in which $f(\underline{v})$ is rotationally invariant, e.g. a Maxwell distribution. The arguments we give also apply to general distributions, considered in Sect. 6. We now place at time $t=0$ a heavy ball of radius $r$ and mass $M$ into this ideal gas system. The initial position $\underline{X}$ and velocity $V$ of the center of the ball may be choosen arbitrarily.

The ball undergoes elastic collisions with the gas particles. The problem we will be concerned with is to describe the motion of the ball in the Brownian limit (BL), where $m \rightarrow 0$ and the gas has density $\lambda \sim m^{-1 / 2}$ and velocity distribution given by $m^{3 / 2} f(\sqrt{m} \underline{v})$. We shall prove that in the BL the velocity $\underline{V}(t)$ of the ball is given by an Ornstein-Uhlenbeck process.

A heuristic central limit theorem type argument for this scaling, which assumes that in the limit the collisions become essentially independent, may be given as follows: When $m \rightarrow 0$ the average energy $m\left\langle v^{2}\right\rangle / 2$ of the gas particles remains constant due to the velocity scaling. Roughly speaking each collision changes the velocity $\underline{V}$ of the heavy particle by $\delta \underline{V} \sim m \underline{v}$ with variance $\left\langle\delta \underline{V}^{2}\right\rangle \sim m$. Hence in a time interval of length $T$ in which there are $N$ collisions the total variance $\langle N\rangle\left\langle\delta \underline{V}^{2}\right\rangle \sim\langle N\rangle m$ will remain constant if $\langle N\rangle \sim r^{2} \lambda T\langle|\underline{v}|\rangle \sim m^{-1}$ or $\lambda \sim m^{-1 / 2}$ for $T$ fixed.

To state our results more precisely we consider the sequence of stochastic processes $V_{m}$, where $V_{m, t}$ is the velocity of the ball at time $t$ in a bath of particles of mass $m$.

Theorem. If $\int|v|^{4} f(\underline{v}) d \underline{v}<\infty$, then the "sequence" $\underline{V}_{m}$ converges in distribution (weakly) to the Ornstein-Uhlenbeck velocity process in the limit $m \rightarrow 0$.

It follows then that the position process $\underline{X}_{m, t}$ converges weakly to the OrnsteinUhlenbeck position process. Because the collision equation is linear in the precollision velocities $\underline{V}, \underline{v}$ and depends only on the ratio $M / m$, the BL is equivalent to a different limit in which $M \rightarrow \infty, m, \lambda$ and the velocity distribution remain constant, the time is scaled like $M$ and space like $\sqrt{M}$. Our theorem thus yields an Ornstein-Uhlenbeck limit for the scaled process $V_{M}(t)=\sqrt{M} \underline{V}(M t)$, for which process the limit theorems in the papers mentioned above were given.

The outline of the paper is as follows: In Sect. 1 we describe the model more precisely. Section 2 presents the main result, for the case when the velocity distribution of the light particles is spherically symmetric, which is proven in 
Sects. 3-5. The latter section is the heart of the matter - showing that the mechanical motion can be approximated by a Markov process when $m \rightarrow 0$. Section 6 generalizes the result to non-isotropic velocity distributions of the light particles; the friction and diffusion coefficient are now tensors. The appendix contains some technical details.

We have also extended our work to the case where the massive particle is not a ball but has a general convex shape. The motion is now one of rotation and translation. We derive the appropriate Ornstein-Uhlenbeck process and write down the corresponding Fokker-Planck equation for this system. This may be used to model the motion of a large molecule in a fluid and will be published separately.

\section{The Mechanical Model}

Throughout this paper we will call the heavy particle the molecule and the light particles atoms or bath particles. Let $\Gamma=\mathbb{R}^{3} \times \mathbb{R}^{3}$ denote the one particle phase space, $\mathscr{B}(\Gamma)$ its Borel algebra and $\mu_{m}$ an absolutely continuous measure on $\Gamma$, defined by

$$
d \mu_{m}=\lambda_{m} d \underline{q} f_{m}(v) d \underline{v}, \underline{q}, \underline{v} \in \mathbb{R}^{3}, \quad v=|\underline{v}|,
$$

where

$$
\lambda_{m}=\lambda / \sqrt{m}, \quad \lambda>0
$$

and

$$
f_{m}(v)=m^{3 / 2} f(\sqrt{m} v)
$$

$f(v)$ is the density of a rotationally invariant probability measure. We assume that the velocity distribution has at least four moments i.e.

$$
\int v^{4} f(v) d \underline{v}<\infty .
$$

The ideal gas of atoms with masses $m$ is then described by a Poisson field $\left(\Omega, \mathscr{F}, \mathbb{P}_{m}\right)$ built on $\left(\Gamma, \mathscr{B}(\Gamma), \mu_{m}\right):$ If $N_{B}=$ the number of particles with coordinates $(\underline{q}, \underline{v})$ in $B \in \mathscr{B}(\Gamma)$, then

$$
\mathbb{P}_{m}\left(\left\{\omega \in \Omega / N_{B}(\omega)=k\right\}\right)=\exp \left(-\mu_{m}(B)\right) \frac{\mu_{m}(B)^{k}}{k !}
$$

It follows that if $B_{1} \ldots B_{l}$ are pairwise disjoint sets the random variables $N_{B_{l}}$ are independent [5]. Here $\omega$ represents a configuration of countably many bath particles i.e. $\omega=\left(\underline{q}_{i}, \underline{v}_{i}\right)_{i \in \mathbb{N}}$.

Remark. We can think of the Poisson field as describing atoms independently distributed in position space with density $\lambda_{m}$ having independent velocities with distribution given by $f_{m}(v)$. For the Maxwell distribution

$$
f_{m}^{M a}(v)=\left(\frac{m \beta}{2 \pi}\right)^{3 / 2} \exp \left(-\beta m v^{2} / 2\right), \quad \beta>0 .
$$


Let us now put the molecule, a ball of radius $r$ and mass $M$, at a position $\underline{X}^{0}$ with velocity $\underline{V}^{0}$. In so doing we remove all atoms from the configuration which are inside the region to be occupied by the molecule.

To describe a collision between the molecule with velocity $\underline{V}$ and an atom with velocity $\underline{v}$, we use the projection $\underline{v}_{n}$ of $\underline{v}$ on the line through the center of the molecule and the collision point on the surface of the molecule. Writing $\underline{v}_{t}$ for the tangential component, this gives

$$
\underline{v}=\underline{v}_{n}+\underline{v}_{t}
$$

and similarly

$$
\underline{V}=\underline{V}_{n}+\underline{V}_{t}
$$

Since the collisions are without friction, conservation of momentum and energy then yields for the post collision velocities $\underline{V}^{\prime}, \underline{v}^{\prime}$

$$
\begin{aligned}
& \underline{V}_{t}^{\prime}=\underline{V}_{t}, \quad \underline{v}_{t}^{\prime}=\underline{v}_{t}, \\
& \underline{v}_{n}^{\prime}=-\frac{M-m}{M+m} \underline{v}_{n}+\frac{2 M}{M+m} \underline{V}_{n}, \\
& \underline{V}_{n}^{\prime}=\frac{M-m}{M+m} \underline{V}_{n}+\frac{2 m}{M+m} \underline{v}_{n} .
\end{aligned}
$$

For $\omega \in \Omega$ we define the velocity $V_{m, t}(\omega)$ of the molecule as a right continuous function of $t$ : If the molecule starts with velocity $\underline{V}^{0}$ then $V_{m, t}(\omega)=\underline{V}^{0}$ for $t<\tau_{1}(\omega)$, the time of the first collision. The velocity changes during this collision according to (1.8)-(1.10) and afterwards the molecule moves freely with the new velocity $\underline{V}^{1}$, until the next collision at $\tau_{2}(\omega)$, i.e. $V_{m, t}(\omega)=\underline{V}^{1}, \tau_{1}(\omega) \leqq t<\tau_{2}(\omega)$, etc. Infinitely many collisions in a finite amount of time, as well as simultaneous collisions of two or more atoms with the molecule (multiple collisions) are problematical. We show in the Appendix that they can be ignored.

For any $I=[0, T], 0<T<\infty$, let $D(I)$ denote the space of right continuous functions with left limits defined on $I$ and $\mathscr{B}(D(I))$ the Borel algebra for the Skorohod-topology [6]. The above description defines a stochastic process $\underline{V}_{m, t}$, $t \in I$, on $\left(\Omega, \mathscr{F}, \mathbb{P}_{m}\right)$, which we may regard as a $D(I)$ valued random variable $\underline{V}_{m}: \Omega \rightarrow D(I)$. Thus $\underline{V}_{m}(\omega)=\underline{V}_{m}(\omega, \cdot)=\underline{V}_{m,}(\omega)$.

$V_{m}$ induces a measure $P_{m}$ on $D(I)$ :

$$
P_{m}(A)=\mathbb{P}_{m}\left(\left\{\omega \mid V_{m}(\omega) \in A\right\}\right),
$$

for all $A \in \mathscr{B}(D(I))$. (Throughout this paper the letter $I$ denotes an arbitrary interval $[0, T], 0<T<\infty$. With slight abuse of notation, we do not distinguish between the induced measures for distinct $I$. We also denote by $P_{m}$ the measure induced on $D([0, \infty))$.)

Remark. The process $\underline{V}_{m}$ is not a Markov process. The molecule can catch up with "slow" atoms with which it has collided earlier and hence are carrying information of past events. These recollisions may be real or virtual. The latter correspond to collisions which are impossible if the past history of the molecule is known. Thus $\underline{V}_{m}$ has a memory. 


\section{The Main Result}

Definition 2.1. The Ornstein-Uhlenbeck velocity process $\underline{V}_{0}$ (with state space $\mathbb{R}^{3}$ ), defined on some probability space $\left(\Omega_{0}, \mathscr{F}_{0}, \mathbb{P}_{0}\right)$, is the Markov (diffusion) process given by the stochastic differential equation $(0.2)$. Its transition probability is

$$
Q_{t}\left(\underline{V}^{0}, d \underline{V}\right)=(2 \pi \delta(t))^{-3 / 2} \exp \left(-\frac{\left(\underline{V}-\alpha(t) \underline{V}^{0}\right)^{2}}{2 \delta^{2}(t)}\right) d \underline{V},
$$

where $\delta^{2}(t)=\frac{D(1-\exp (-2 a t))}{2 a}, \alpha(t)=\exp (-a t)$

$V_{0}$ induces the measure $P_{0}$ on $D(I)$, i.e.

$$
P_{0}(A)=\mathbb{P}_{0}\left(\left\{\omega \in \Omega_{0} / V_{0}(\omega) \in A\right\}\right)
$$

for all $A \in \mathscr{B}(D(I))$.

Definition 2.2. Let $P_{n}, P$ be probability measures on $(S, \mathscr{B}(S))$ where $S$ is a metric space and $\mathscr{B}(S)$ the Borel algebra. Then $P_{n}$ converges weakly to $P\left(P_{n} \Rightarrow P\right)$ if for all bounded continuous real functions $h$ on $S \lim _{n \rightarrow \infty} \int h d P_{n}=\int h d P$ or, equivalently, if $\lim \inf P_{n}(G) \geqq P(G)$ for all open $G[6]$.

Note that "weak convergence" depends on the topology of $S$. The following theorem is concerned with weak convergence in the sense of the Skorohodtopology, which does not in general imply weak convergence in the sense of the uniform topology [6].

We set

$$
\Phi_{i}=\frac{1}{2} \int\left|v_{x}\right|^{i} f(v) d \underline{v}, \quad i=1, \ldots, 4,
$$

where $\underline{v}=\left(v_{x}, v_{y}, v_{z}\right)$.

Our main result is

Theorem 2.1. For any $I, P_{m} \underset{m \rightarrow 0}{\Rightarrow} P_{0}$ on $D(I)$, where $P_{0}$ corresponds to the OrnsteinUhlenbeck process with $\underline{V}_{0}(0)=\underline{V}^{0}$ and with parameters

$$
a=\frac{16 \pi}{3} \lambda \frac{r^{2}}{M} \Phi_{1}, \quad D=\frac{16 \pi}{3} \lambda \frac{r^{2}}{M^{2}} \Phi_{3} .
$$

Remark. The convergence described in Theorem 2.1 can be expressed in terms of the processes $V_{m}$ and $V_{0}$ as " $V_{m}$ converges in distribution to $V_{0}$ ", denoted by $\underline{V}_{m} \stackrel{\vartheta}{\Rightarrow} \underline{V}_{0}$.

Remark. Letting $t \rightarrow \infty$ in (2.1) we get the density of the stationary probability distribution of $\underline{V}_{0}: p_{s t} \sim \exp \left(-\underline{V}^{2} a / D\right)$ which determines the "temperature" of the molecule:

$$
\beta_{M}=2 a / D M=2 \Phi_{1} / \Phi_{3}
$$

For the Maxwell distribution $\Phi_{1}^{\mathrm{Max}}=(2 \beta \pi)^{-1 / 2}, \Phi_{3}^{\mathrm{Max}}=\left(2 / \pi \beta^{3}\right)^{1 / 2}$ and hence $\beta_{M}^{\mathrm{Max}}=\beta$, i.e. the molecule is in thermodynamic equilibrium with the bath. It is not 
difficult to see that other distributions would in general lead to different temperatures i.e. $M\left\langle V^{2}\right\rangle \neq m\left\langle v^{2}\right\rangle$. The drift and diffusion coefficients in the case of the Maxwell distribution are [11]

$$
a^{\operatorname{Max}}=\frac{8}{3}\left(\frac{2 \pi}{\beta}\right)^{1 / 2} \lambda \frac{r^{2}}{M}, \quad D^{\operatorname{Max}}=\frac{16}{3}\left(\frac{2 \pi}{\beta^{3}}\right)^{1 / 2} \lambda \frac{r^{2}}{M^{2}} .
$$

Concerning the position process $\underline{X}_{m}$ we have

Corollary 2.1. $\underline{X}_{m} \stackrel{\vartheta}{\Rightarrow} \underline{X}_{0}$, where $\underline{X}_{0, t}=\int_{0}^{t} \underline{V}_{0, s} d s+\underline{X}^{0}$.

Proof. Define $K: D(I) \rightarrow C(I)$, the space of continuous functions on $I$, by

$$
K \underline{y}(t)=\int_{0}^{t} \underline{y}(s) d s+\underline{X}^{0}, \quad \underline{y} \in D(I) .
$$

$K$ is a continuous map from $D(I)$ into $C(I)$. Let $g$ be any bounded continuous real function on $C(I)$. With $P_{m}^{K}, P_{0}^{K}$ the induced measures corresponding to $\underline{X}_{m}, \underline{X}_{0}$ we have, by Theorem 2.1,

$$
\lim _{m \rightarrow 0} \int g(\underline{x}) d P_{m}^{K}(\underline{x})=\lim _{m \rightarrow 0} \int g \circ K(\underline{y}) d P_{m}(\underline{y})=\int g \circ K(\underline{y}) d P_{0}(\underline{y})=\int g(\underline{x}) d P_{0}^{K}(\underline{x}),
$$

since $g \circ K$ is a bounded continuous function on $D(I)$.

Since the mechanical process $V_{m}$ is not Markovian, Theorem 2.1 is concerned with the weak convergence of non Markovian processes to a Markov process. The proof we give consists of two steps. First we consider a modification of the mechanical process, an abstract Markov process $\tilde{V}_{m}$, for which we prove

$$
\tilde{V}_{m} \stackrel{\vartheta}{\Rightarrow} \underline{V}_{0} .
$$

In the second step we establish the closeness of the paths of a suitable realization $V_{m}^{\prime}$ of $\tilde{V}_{m}$ to the paths of $V_{m}$ as $m$ goes to zero. It is indeed the central part of this work to find a "mechanical version" $\underline{V}_{m}^{\prime}$ of a Markov process $\tilde{V}_{m}$ converging to $\underline{V}_{0}$ which is close to the true mechanical process $\underline{V}_{m}$ in the following sense: $\underline{V}_{m}^{\prime}$ and $\underline{V}_{m}$ are realized on the same probability space $\left(\overline{\bar{\Omega}}, \overline{\mathscr{F}}, \overline{\mathbb{P}}_{m}\right)$ in such a way that for all $\varepsilon>0$ and any $I$

$$
\lim _{m \rightarrow 0} \overline{\mathbb{P}}_{m}\left(\left\{\bar{\omega} \in \bar{\Omega} / \sup _{t \in I}\left|\underline{V}_{m}^{\prime}(\bar{\omega}, t)-\underline{V}_{m}(\bar{\omega}, t)\right| \geqq \varepsilon\right\}\right)=0 .
$$

Theorem 2.1 easily follows from (2.5) and (2.6) [6].

\section{The Markov Approximation}

Let us consider the mechanical process $\underline{U}_{m}$ in which only collisions with "fast" atoms, namely with precollision velocity $\underline{v}$ satisfying $\left|\underline{v}_{n}\right| \geqq c_{m}=m^{-1 / 5}$, have an effect on the motion of the molecule; in other words collisions of "slow" atoms $\left(\left|\underline{w}_{n}\right|<c_{m}\right)$ "don't count". Until $\left|\underline{U}_{m, t}(\cdot)\right| \geqq c_{m}$ any fast atom which collides with the molecule cannot have collided earlier, as may be seen by tracing the paths of the atom and the molecule from the collision point backwards in time. Thus all atoms 
which recollide with the molecule are slow and by our rules they have no effect. Consequently the process $\underline{U}_{m}$ has no memory until $\left|\underline{U}_{m, t}(\cdot)\right| \geqq c_{m}$. We shall now define a Markov process $\underline{V}_{m}$ based on these rules for collisions. We will use an informal definition of the events in which we are interested, leaving a precise mathematical definition to the reader.

We define $v_{n}, V_{n} \in \mathbb{R}$ by

$$
\underline{v}_{n}=v_{n} \underline{e}_{n}, \quad \underline{V}_{n}=V_{n} \underline{e}_{n},
$$

where $\underline{e}_{n}$ is directed towards the molecule. Let $\vartheta \in[0, \pi]$ be given by

$$
\cos \vartheta=\underline{e} \cdot \underline{e}_{n},
$$

where $\underline{e}$ is the unit vector of some fixed direction in space, and let $\varphi \in[0,2 \pi)$ denote an azimuthal angle, so that

$$
d \underline{S}=-r^{2} d \Omega \underline{e}_{n}=-r^{2} \sin \vartheta d \vartheta d \varphi \underline{e}_{n}
$$

is a surface element of the molecule. As a consequence of the translation invariance of the Poisson field we have

Lemma 3.1. Suppose that at time the molecule has velocity $\underline{V}$ and is surrounded by a bath of atoms having the Poisson distribution described in (1.4). The probability $p_{m}(d t, d \underline{v}, d \Omega, \underline{V})$ for the collision of an atom with velocity $\underline{v} \in d \underline{v}$ with the molecule in a surface element $d \underline{S}=-r^{2} d \Omega \underline{e}_{n}$ in $[t, t+d t]$ is given by

$$
p_{m}(d t, d \underline{v}, d \Omega, \underline{V})=\lambda_{m} r^{2} d \Omega\left(v_{n}-V_{n}\right)_{+} d t f_{m}(v) d \underline{v},
$$

where $\left(v_{n}-V_{n}\right)_{+}=\max \left(v_{n}-V_{n}, 0\right)$.

Proof. For the occurrence of the collision, an atom with velocity $\underline{v} \in d \underline{v}$, has to be in a volume element $r^{2} d \Omega\left(v_{n}-V_{n}\right)_{+} d t d \underline{v}$ of the phase space, whose measure in view of (1.1) is given by (3.4).

It is convenient to integrate (3.4) over the tangential velocity components, so that we obtain the probability for a "collision with normal velocity $v_{n} \in d v_{n}$ ",

$$
p_{m}\left(d t, d v_{n}, d \Omega, \underline{V}\right)=\lambda_{m} r^{2} d \Omega\left(v_{n}-V_{n}\right)_{+} d t f^{1}\left(v_{n}\right) d v_{n},
$$

where $f^{1}$ denotes the density of the one dimensional marginal distribution of the velocities of the atoms.

We set

$$
N_{m}(\underline{V})=\int d \Omega \int_{c_{m}}^{\infty}\left(v_{n}-V_{n}\right)_{+} f_{m}^{1}\left(v_{n}\right) d v_{n},
$$

where $d \Omega$ is defined as in (3.3), and let

$$
g_{m}\left(\underline{V}, \underline{v}_{n}\right)= \begin{cases}\frac{1}{N_{m}(\underline{V})}\left(v_{n}-V_{n}\right)_{+} f_{m}^{1}\left(v_{n}\right), & v_{n} \geqq c_{m} \\ 0, & v_{n}<c_{m} .\end{cases}
$$

A simple calculation shows that $N_{m}(\underline{V})=N_{m}(0)$ for $|\underline{V}| \leqq c_{m}$. Setting

$$
\bar{\lambda}_{m}=\lambda_{m} r^{2} N_{m}(0)
$$


we define a probability for "collisions" with normal velocities $\underline{v}_{n},\left|\underline{v}_{n}\right| \geqq c_{m}$,

$$
\bar{p}_{m}\left(d t, d v_{n}, d \Omega, \underline{V}\right)=\bar{\lambda}_{m} d t d \Omega g_{m}\left(\underline{V}, \underline{v}_{n}\right) d v_{n},
$$

which equals (3.5) for $|\underline{V}| \leqq c_{m}$.

Using the collision Eqs. (1.8)-(1.10), $g_{m}\left(\underline{V}, \underline{v}_{n}\right) d v_{n} d \Omega$ can be transformed into a transition kernel $G_{m}(\underline{V}, d \underline{V})$, giving the jump probability from $\underline{V}$ to $\underline{V}^{\prime} \in d \underline{V}$ in one "collision".

Definition 3.1. Let $\tilde{V}_{m}$ be the Poisson jump process, defined on the probability space $\left(\tilde{\Omega}, \tilde{\mathscr{F}}, \tilde{\mathbb{P}}_{m}\right)$, with mean waiting time $\bar{\lambda}_{m}^{-1}$ and transition kernel $G_{m}(\underline{V}, d \underline{V})$. Let $\tilde{P}_{m}$ denote the measure induced by $\tilde{V}_{m}$ on $(D(I), \mathscr{B}(D(I)))$.

In the next section we shall prove

Lemma 3.2. $\tilde{V}_{m} \stackrel{\vartheta}{\Rightarrow} V_{m}$.

Remark 3.1. Note that (3.5) is the probability for collisions with fast atoms in the process $\underline{U}_{m}$ until $\left|\underline{U}_{m, t}\right| \geqq c_{m}$; thus the measure $P_{m}^{U}$ on $D(I)$ induced by $\underline{U}_{m}$ agrees with $\tilde{P}_{m}$ on the set

$$
H_{m}=\left\{\underline{x} \in D(I) / \sup _{t \in I}|\underline{x}|<c_{m}\right\}
$$

which is open in $D(I)$. From this it follows easily that $\underline{U}_{m} \stackrel{\vartheta}{\Rightarrow} \underline{V}_{0}$, by noting that for every open set $G \subset D(I)$

$$
P_{m}^{U}(G) \geqq P_{m}^{U}\left(G \cap H_{m}\right)=\tilde{P}_{m}\left(G \cap H_{m}\right) \geqq \tilde{P}_{m}\left(G \cap H_{n}\right), \quad n \geqq m
$$

and by Lemma 3.2

$$
\liminf _{m \rightarrow 0} P_{m}^{U}(G) \geqq \liminf _{m \rightarrow 0} \tilde{P}_{m}\left(G \cap H_{n}\right) \geqq P_{0}\left(G \cap H_{n}\right) .
$$

Since this is true for all $n$, we can take $n \rightarrow \infty$, to obtain

$$
\liminf P_{m}^{U}(G) \geqq P_{0}(G),
$$

from which the result follows (Definition 2.2).

Remark 3.2. The reason for using the Markov process defined by $\bar{p}$ in (3.9) rather then the "more natural" one described by (3.5) is mainly technical, e.g. the details in Sect. 4 are easier to handle.

\section{Proof of Lemma 3.2}

We shall use some facts from the theory of probability semigroups. Let $\underline{Z}$ denote a Markov process on $D(I)$ having transition probability $Q_{t}(\underline{x}, d \underline{y})$. To $\underline{Z}$ corresponds a contraction semigroup $T_{t}$ defined on $B$, the Banach space of bounded measurable functions $h: \mathbb{R}^{3} \rightarrow \mathbb{R}$, with the sup-norm $\|\cdot\|$,

$$
T_{t} h(\underline{x})=\int_{R^{3}} h(\underline{y}) Q_{t}(\underline{x}, d \underline{y}) .
$$

Let $C_{0}$ denote the Banach subspace of $B$ consisting of continuous functions vanishing at $\infty$. Suppose $C_{0}$ is invariant under $T_{t}$ and $T_{t}$ on $C_{0}$ is strongly 
continuous: $T_{t} C_{0} \subseteq C_{0}$ and $\lim _{t \rightarrow 0}\left\|T_{t} h-h\right\|=0$ for all $h \in C_{0}$. Then $\underline{Z}$ is called a Markov- $C_{0}$-process [7].

The (infinitesimal) generator $A$ of $\underline{Z}$ is given by

$$
A h=\lim _{t \rightarrow 0} \frac{T_{t} h-h}{t}
$$

on the domain $\Delta(A)$ consisting of all $h \in C_{0}$ for which the limit exists in the supnorm topology.

Remark. A diffusion process is a Markov- $C_{0}$-process. The restriction of the generator $A$ of a diffusion process to $C_{c}^{\infty}$, the set of infinitely differentiable functions of compact support, is an elliptic differential operator. For the generator $A^{(0)}$ of the Ornstein-Uhlenbeck process $\underline{V}_{0}$ we have

$$
\left.A^{(0)}\right|_{C_{c}^{\infty}}=-a \underline{V} \cdot \nabla+\frac{1}{2} D \nabla \cdot \nabla, \quad \nabla=\text { gradient },
$$

where $a, D$ are given by (2.4).

Lemma 4.1. Consider a sequence $\underline{Z}_{n}$ of Markov processes with sample paths in $D(I)$ and generators $A^{(n)}$. Suppose $\underline{Z}$ is a Markov- $C_{0}$-process with sample paths in $D(I)$ and generator $A$. Let $K$ be a core for $A$ and suppose that $h \in K$ implies that $h \in \Delta\left(A^{(n)}\right)$ for all sufficiently large $n$. Suppose that the initial distributions of $\underline{Z}_{n}$ converge weakly to the initial distribution of $\underline{Z}$ and that

$$
\lim _{n \rightarrow \infty} \sup _{\underline{x} \in \mathbb{R}^{3}}\left|A^{(n)} h(\underline{x})-A h(\underline{x})\right|=0
$$

for all $h \in K$. Then

$$
\underline{Z}_{n} \underset{n \rightarrow \infty}{\stackrel{\vartheta}{\longrightarrow}} \underline{Z}
$$

Remark. A core for $A$ is a linear subspace $K$ of $\Delta(A)$, such that $A$ is the closure of the restriction of $A$ to $K$. $K$ is a core if, for example, $K$ is dense and $T_{t} K \subseteq K$ [8]. For the generator of a diffusion process cores can be found using regularity properties of the solutions of the parabolic equation for the transition density. For example in the case of the Ornstein-Uhlenbeck process one can conclude that $T_{t} C_{0}^{2} \subseteq C_{0}^{2}$, where $C_{0}^{2}$ denotes the set of twice continuously differentiable functions vanishing at infinity together with the first and second order derivatives. Since $C_{0}^{2}$ is dense in $C_{0}, C_{0}^{2}$ is a core. It then easily follows that $C_{c}^{\infty}$ is also a core.

Remark. Lemma 4.1 is stated for a slightly more general situation in a paper by Kurtz [8]. Similar results have been given earlier by Skorohod [9]. Conditions for a set to be a core and its role in the convergence of semigroups can be found in [8] and in [10].

We now turn to the proof of Lemma 3.2. Let $\tilde{A}^{(m)}$ be the generator and $\tilde{T}_{t}^{(m)}$ the semigroup of $\tilde{\underline{V}}_{m}$. By Lemma 4.1 it suffices to show that

$$
\lim _{m \rightarrow 0}\left\|\tilde{A}^{(m)} h-A^{(0)} h\right\|=0, \quad \text { for } h \in C_{c}^{\infty} .
$$

Let us denote by $\tilde{E}_{V^{0}}^{(m)}(\cdot)=\tilde{E}^{(m)}\left(\cdot \mid \tilde{V}_{m}(0)=\underline{V}^{0}\right)$ the expectation for the process $\tilde{V}_{m}$ starting at $\underline{V}^{0}$. Then

$$
\tilde{T}_{t}^{(m)} h\left(\underline{V}^{0}\right)=\tilde{E}_{V^{0}}^{(m)}\left(h\left(\tilde{V}_{m, t}\right)\right) \quad \text { for } \quad h \in B
$$


and

$$
\tilde{A}^{(m)} h\left(\underline{V}^{0}\right)=\lim _{t \rightarrow 0} \frac{1}{t}\left(\tilde{E}_{V^{0}}^{(m)}\left(h\left(\tilde{V}_{m, t}\right)\right)-h\left(\underline{V}^{0}\right)\right) \quad \text { for } \quad h \in \Delta\left(\tilde{A}^{(m)}\right) .
$$

For the case of a Poisson jump process this becomes very simple, since the probability for more than one jump in time $t$ is of order $O\left(t^{2}\right)$. Hence in the above expectation we only have to consider terms involving no more than one jump. We obtain, according to Definition 3.1,

$$
\begin{aligned}
\tilde{A}^{(m)} h\left(\underline{V}^{0}\right) & =\lim _{t \rightarrow 0} \frac{1}{t}\left(e^{-\bar{\lambda}_{m} t} h\left(\underline{V}^{0}\right)+e^{-\bar{\lambda}_{m t}} \bar{\lambda}_{m} t \int G_{m}\left(\underline{V}^{0}, d \underline{V}\right) h(\underline{V})-h\left(\underline{V}^{0}\right)\right. \\
& =-\bar{\lambda}_{m} h\left(\underline{V}^{0}\right)+\bar{\lambda}_{m} \int G_{m}\left(\underline{V}^{0}, d \underline{V}\right) h(\underline{V}) .
\end{aligned}
$$

Note that $\Delta\left(\tilde{A}^{(m)}\right)=C_{0} \supset C_{c}^{\infty}$.

In view of (1.10), we set $\underline{V}-\underline{V}^{0}=q_{m} \underline{u}_{n}$, where

$$
q_{m}=2 m /(M+m)
$$

and $\underline{u}_{n}=\underline{v}_{n}-\underline{V}_{n}^{0}$. A Taylor expansion for $h$ around $\underline{V}^{0}$ yields

$$
h\left(\underline{V}^{0}+q_{m} \underline{u}_{n}\right)=h\left(\underline{V}^{0}\right)+q_{m} \underline{u}_{n} \cdot \nabla h\left(\underline{V}^{0}\right)+(1 / 2) q_{m}^{2}\left(u_{n} \cdot \nabla\right)^{2} h\left(\underline{V}^{0}\right)+(1 / 6) q_{m}^{3}\left(\underline{u}_{n} \cdot \nabla\right)^{3} h\left(\underline{V}^{\prime}\right),
$$

where $\underline{V}^{\prime}=\underline{V}^{0}+\gamma q_{m} \underline{u}_{n}, \gamma \in[0,1]$.

Introducing this into (4.4) the integral becomes, with

$$
\underline{v}_{n}-\underline{V}_{n}^{0}=\left(v_{n}-V_{n}^{0}\right)_{+} \underline{e}_{n},
$$

and after replacing $G_{m}(\underline{V}, d \underline{V})$ by $g_{m}\left(\underline{V}, \underline{v}_{n}\right) d v_{n} d \Omega$, a sum of four integrals of the form

$$
J_{i}=\frac{\bar{\lambda}_{m}}{N_{m}\left(\underline{V}^{0}\right)} \int d \Omega \int_{c_{m}}^{\infty}\left(v_{n}-V_{n}^{0}\right)_{+}^{i} \frac{\left(q_{m} \underline{e}_{n} \cdot \nabla\right)^{i-1}}{(i-1) !} h\left(\underline{V}_{i}\right) f_{m}^{1}\left(v_{n}\right) d v_{n},
$$

where $\underline{V}_{i}=\underline{V}^{0}$ for $i=1,2,3$ and $V_{4}=V^{\prime}$.

In the computations we shall use the fact that for $i=1, \ldots, 4$

$$
\lim _{m \rightarrow 0} m^{i / 2} \int_{c_{m}}^{\infty} x^{i} f_{m}^{1}(x) d x=\lim _{m \rightarrow 0} \int_{c_{m} \sqrt{m}}^{\infty} y^{i} f^{1}(y) d y=\Phi_{i} .
$$

Since $h \in C_{c}^{\infty}$, we may assume $h(x)=0$ for $|x|>b$ and we choose $m$ so small that $c_{m}>b$.

We start by showing that $J_{4}$ goes to zero uniformly in $\underline{V}^{0}$ as $m$ goes to zero.

For $V^{0} \leqq c_{m}, J_{4} \leqq O\left(m^{1 / 2}\right)$ since $\int_{c_{m}}^{\infty} y^{4} f_{m}^{(1)}(y) d y \leqq m^{-2} \Phi_{4}$,

$$
\left(\bar{\lambda}_{m} / N_{m}\left(\underline{V}^{0}\right)\right)=\lambda_{m} r^{2} \sim m^{-1 / 2}, \quad \sup _{\underline{e}_{n}, \underline{V}^{\prime} \in \mathbb{R}^{3}}\left|\left(\underline{e}_{n} \cdot \nabla\right)^{3} h\left(\underline{V}^{\prime}\right)\right|<\infty, \quad \text { and } \quad q_{m}^{3} \sim m^{3} .
$$

[Expressions of the form $O\left(m^{k}\right)$ denote quantities depending perhaps on $h$ but not on $\underline{V}^{0}$.]

For $V^{0} \geqq c_{m}$ we are in the complement of the support of $h$. Since $\underline{V}^{\prime}=\underline{V}^{0}$ $+\gamma q_{m}\left(\underline{v}_{n}-\underline{V}_{n}^{0}\right), \underline{v}_{n}$ must be such that for the post collision velocity $\underline{V}$ we have $|\underline{V}|<b$, and the "easiest" way for this to occur is if $\underline{V}^{0}=-V^{0} \underline{e}_{n}$. A simple calculation using 
(1.10) shows that $\mid \underline{V \mid \leqq}$ implies that for $m$ sufficiently small

$$
\left|\underline{v}_{n}\right| \geqq-b(M+m) / 2 m+V^{0}(M-m) / 2 m>V^{0}
$$

for all $V^{0} \geqq c_{m}$. Thus $J_{4}$ can be estimated as follows :

$$
J_{4} \leqq \frac{1}{6} q_{m}^{3} \frac{\bar{\lambda}_{m}}{\min _{\underline{V}^{0} \in \mathbb{R}^{3}} N_{m}\left(\underline{V}^{0}\right)} 4 \pi \sup _{\underline{e}_{n}, \underline{V}^{\prime} \in \mathbb{R}^{3}}\left|\left(\underline{e}_{n} \cdot \nabla\right)^{3} h\left(V^{\prime}\right)\right| \int_{V^{0}}^{\infty}\left(v_{n}+V^{0}\right)^{4} f_{m}^{(1)}\left(v_{n}\right) d v_{n} \leqq O\left(m^{1 / 2}\right),
$$

since $N_{m}\left(\underline{V}^{0}\right) \geqq N_{m}(0)$.

For $J_{3}$ we observe that

$$
\begin{aligned}
J_{3}= & \frac{1}{2} q_{m}^{2} \lambda_{m} r^{2} \int_{c_{m}}^{\infty} v_{n}^{3} f_{m}^{1}\left(v_{n}\right) d v_{n} \nabla \cdot\left(\int d \Omega \underline{e}_{n} \underline{e}_{n}\right) \cdot \nabla h\left(\underline{V}^{0}\right)+O(m) \\
& \underset{m \rightarrow 0}{\longrightarrow} \frac{8 \pi \lambda r^{2}}{3 M^{2}} \Phi_{3} \nabla \cdot \nabla h\left(\underline{V}^{0}\right),
\end{aligned}
$$

uniformly in $V^{0}$, since

$$
\int d \Omega \underline{e}_{n} \underline{e}_{n}=\frac{4 \pi}{3} \mathbb{1}
$$

$\left(\mathbb{1}=3 \times 3\right.$ identity; trace of tensor product $\underline{e}_{n} \underline{e}_{n}=1$.)

For $J_{2}$ we obtain

$$
\begin{aligned}
J_{2}= & q_{m} \lambda_{m} r^{2}\left[\int_{c_{m}}^{\infty} v_{n}^{2} f_{m}^{1}\left(v_{n}\right) d v_{n} \int d \Omega \underline{e}_{n} \cdot \nabla h\left(\underline{V}^{0}\right)-2 \int_{c_{m}}^{\infty} v_{n} f_{m}^{1}\left(v_{n}\right) d v_{n} \underline{V}^{0} \cdot \int d \Omega \underline{e}_{n} \underline{e}_{n} \cdot \nabla h\left(\underline{V}^{0}\right)\right] \\
& +O\left(m^{1 / 2}\right) \underset{m \rightarrow 0}{\longrightarrow}-\frac{16 \pi \lambda r^{2}}{3 M} \Phi_{1} \underline{V}^{0} \cdot \nabla h\left(\underline{V}^{0}\right),
\end{aligned}
$$

uniformly in $\underline{V}^{0}$, since $\int d \Omega \underline{e}_{n}=0$.

We complete the proof of Lemma 3.2 by noting that in view of (3.6) $J_{1}$ cancels the first term on the r.h.s. of (4.4).

\section{The Closeness of the Paths}

Throughout this section we set $M=1, \lambda=1, r=1$ to simplify the notation. Our aim is to define a realisation $\underline{V}_{m}^{\prime}$ of the Markov process $\tilde{V}_{m}$ which is close [in the sense of (2.6)] to the true mechanical process. We will call a simultaneous realisation (coupling) of both processes for which (2.6) is true, a good coupling of $\underline{V}_{m}$ and $\underline{V}_{m}$. There exist many couplings of $\tilde{V}_{m}$ and $\underline{V}_{m}$. One natural choice is the process $\underline{U}_{m}$, which is naturally defined on $\Omega$ by the prescription of not counting collisions with slow atoms, and by Remark $3.1 \underline{U}_{m} \stackrel{\vartheta}{\Rightarrow} \underline{V}_{0}$. Actually a similar coupling was used by Holley in the one dimensional case, but in higher dimensions this coupling is not as useful for the following reason: The effect of a collision in higher dimensions involves the impact parameter of the collision, i.e. it depends on the collision point on the surface of the molecule. If we consider now two identical copies of a bath 
configuration and compare the motion of the "Markov molecule" in the $\underline{U}_{m}$-process with the motion of the "mechanical molecule" in the $\underline{V}_{m}$-process under the same initial conditions, their velocities will differ after some time due to collisions with slow atoms in the $\underline{V}_{m}$-process. Hence after some time the molecules will be in different positions. Then a fast atom which collides with the molecule in both cases, will collide at different points on the surface of the molecule (as well as at different times) and hence with different normal velocities. A rough estimate shows that this effect will cause an error in the velocities which is not sufficiently controllable to show (2.6).

We obtain a good coupling by constructing $\underline{V}_{m}^{\prime}$ in such a way that collisions with fast atoms will "usually" occur at the same time and at the same point as in the actual mechanical process $\underline{V}_{m}$.

Recall that the Markov process $\tilde{V}_{m}$ is a Poisson jump process with mean waiting time $\bar{\lambda}_{m}^{-1}$ and jump distribution determined by $g_{m}\left(\underline{V}, \underline{v}_{n}\right) d v_{n} d \Omega$, i.e. it may be characterized by the (collision) rates

$$
r_{m}\left(\underline{V}, \underline{v}_{n}\right)=\bar{\lambda}_{m} g_{m}\left(\underline{V}, \underline{v}_{n}\right) .
$$

By the same argument as given before for the process $\underline{U}_{m}$, we have for the mechanical process $\underline{V}_{m}$ that until $\left|\underline{V}_{m, t}\right| \geqq c_{m}$ a fast atom which collides with the molecule cannot have collided earlier. Hence until $\left|\underline{V}_{m, t}\right| \geqq c_{m}$ the fast atoms with which the molecule collides are all Poisson distributed so that the collisions between the molecule and fast atoms are also governed by the rates (5.1). Note, that due to slow atom collisions (5.1) does not describe the mechanical process even before $\left|\underline{V}_{m, t}\right| \geqq c_{m}$.

In the following we denote by $(\mathrm{Me})$ the mechanical molecule, in the mechanical process $\underline{V}_{m}$, and by (Ma) the Markov molecule, undergoing the Markov process $\underline{V}_{m}^{\prime}$ which we now define. We use $\underline{V}\left(\underline{V}^{\prime}\right)$ as the generic variable for the velocity of $(\mathrm{Me})((\mathrm{Ma}))$. (Me) and (Ma) have the same initial conditions. Given a configuration $\omega$ and thus the motion $\underline{V}_{m, t}(\omega)$ of $(\mathrm{Me})$, we specify the corresponding motion of (Ma) in two steps.

(i) We observe the motion of $(\mathrm{Me})$; when, and only when, $(\mathrm{Me})$ undergoes a collision with a fast atom, with normal velocity $\underline{v}_{n}$, the velocity of $(\mathrm{Ma})$ is changed as if it too had suffered an identical collision, i.e. according to $(1.8,1.10)$ with the same $\underline{v}_{n}$.

Using this prescription the velocities $\underline{V}^{\prime}$ and $\underline{V}$ coincide until (Me) suffers a collision with a slow atom. In particular (until $|\underline{V}| \geqq c_{m}$ ) the collision rate for (Ma) is given by $r_{m}\left(\underline{V}^{\prime}, \underline{v}_{n}\right)=r_{m}\left(\underline{V}, \underline{v}_{n}\right)$. Afterwards the velocities of (Ma) and (Me) will differ, since collisions with slow atoms affect $(\mathrm{Me})$ but not $(\mathrm{Ma})$, and the collision rate for (Ma) will no longer be given by $r_{m}\left(\underline{V}^{\prime}, \underline{v}_{n}\right)$; rather it will continue to be equal to $r_{m}\left(\underline{V}, \underline{v}_{n}\right) \neq r_{m}\left(\underline{V}^{\prime}, \underline{v}_{n}\right)$. Since however we wish $\underline{V}_{m}^{\prime}$ to be a realisation of $\underline{\tilde{V}}_{m}$ it should have rates $r_{m}\left(\underline{V}^{\prime}, \underline{v}_{n}\right)$. This necessitates modifying the prescription given in (i), which we now do.

(ii) To obtain a process with the correct rates we modify (i) by either ignoring some collisions [so that they produce no effect on (Ma)] or adding some "extra collisions", depending on whether $r_{m}\left(\underline{V}^{\prime}, \underline{v}_{n}\right)$ is greater or less than $r_{m}\left(\underline{V}, \underline{v}_{n}\right)$ : Let $\tau=\inf \left\{t \geqq 0 \| V_{m, t} \mid \geqq c_{m}\right\}$. 
(a) The rate for the occurrence of these extra collisions is

$$
R_{m}\left(\underline{V}^{\prime}, \underline{V}, \underline{v}_{n}\right)=\max \left(r_{m}\left(\underline{V}^{\prime}, \underline{v}_{n}\right)-r_{m}\left(\underline{V}, \underline{v}_{n}\right), 0\right) \text { for } t<\tau
$$

and is $r_{m}\left(\underline{V}^{\prime}, \underline{v}_{n}\right)$ for $t \geqq \tau$.

(b) The probability that a collision of (i) counts (i.e. is not ignored) is

$$
p_{m}\left(\underline{V}^{\prime}, \underline{V}, \underline{v}_{n}\right)=\min \left(\frac{r_{m}\left(\underline{V}^{\prime}, \underline{v}_{n}\right)}{r_{m}\left(\underline{V}, \underline{v}_{n}\right)}, 1\right) \text { for } t<\tau
$$

and is 0 for $t \geqq \tau$.

Prescription (i) modified as in (ii), yields a process $V_{m}^{\prime}$ governed by the rates $r_{m}\left(\underline{V}^{\prime}, \underline{v}_{n}\right)$ (which is therefore Markovian).

Note that the realisation $\underline{V}_{m}^{\prime}$ of $\tilde{V}_{m}$ has mechanical as well as purely stochastic aspects. To a path $\underline{V}_{m}(\omega, t)$ of the mechanical process corresponds a set of paths in the $V_{m}^{\prime}$-process whose distribution is determined by the rate for random extracollisions and the probability of collisions counting for the Markov molecule.

Let $\left(\bar{\Omega}, \overline{\mathscr{F}}, \overline{\mathbb{P}}_{m}\right)$ be a probability space on which $\underline{V}_{m}$ and $\underline{V}_{m}^{\prime}$, satisfying the above description, are both realized; in particular $\overline{\mathbb{P}}_{m}\left(\left\{\bar{\omega} \in \bar{\Omega} \mid \underline{V}_{m}^{\prime}(\bar{\omega}) \in A\right\}\right)=\tilde{P}_{m}(A)$, for all $A \in \mathscr{B}(D(I))$ and $V_{m}^{\prime}$ differs from $\underline{V}_{m}$ only by virtue of the rates $R_{m}$ and the probabilities $p_{m}$. [We may think of $\bar{\Omega}$ as a product space $\bar{\Omega}=\Omega \times H$, where the purely stochastic effects are represented by $H$, so that $\underline{V}_{m}(\bar{\omega})=\underline{V}_{m}((\omega, h))=\underline{V}_{m}(\omega)$, for $\bar{\omega}=(\omega, h) \in \bar{\Omega}$, and $\left.\overline{\mathbb{P}}(\cdot \times H)=\mathbb{P}_{m}(\cdot).\right]$

We now have a good coupling.

Lemma 5.1. For any $I$ and for all $\varepsilon>0$

$$
\lim _{m \rightarrow 0} \overline{\mathbb{P}}_{m}\left(\left\{\bar{\omega} \in \bar{\Omega}\left|\sup _{t \in I}\right| \underline{V}_{m, t}^{\prime}(\bar{\omega})-V_{m, t}(\bar{\omega}) \mid \geqq \varepsilon\right\}\right)=0 .
$$

Proceeding as in Holley [2] we first prove

Lemma 5.2. If $t_{0} \geqq 0$ is such that

for all $\varepsilon>0$, then

$$
\lim _{m \rightarrow 0} \overline{\mathbb{P}}_{m}\left(\left\{\sup _{0 \leqq t \leqq t_{0}}\left|\underline{V}_{m, t}^{\prime}-\underline{V}_{m, t}\right| \geqq \varepsilon\right\}\right)=0
$$

$$
\lim _{m \rightarrow 0} \widetilde{\mathbb{P}}_{m}\left(\left\{\sup _{0 \leqq t \leqq t_{0}+z}\left|\underline{V}_{m, t}^{\prime}-\underline{V}_{m, t}\right| \geqq \varepsilon\right\}\right)=0
$$

for all $\varepsilon>0$, where $z=1 /\left(128 \pi \Phi_{1}\right)$.

Remark. Lemma 5.1 follows easily from Lemma 5.2: Since $\underline{V}_{m}^{\prime}(0)=\underline{V}_{m}(0)$, so that for $t_{0}=0$ the hypothesis of Lemma 5.2 is fulfilled, we obtain that (5.4) is satisfied for $I=[0, z]$. Again applying Lemma 5.2 with $t_{0}=z$, we obtain (5.4) for $I=[0,2 z]$. Iterating until $T$ is surpassed we obtain (5.4) for any $I=[0, T]$.

Proof of Lemma 5.2. Fix $\varepsilon>0$ and $t_{0}$ and introduce the stopping time

$$
t_{m}^{*}=\inf _{t \geqq t_{0}}\left\{t:\left|\underline{V}_{m, t}^{\prime}-\underline{V}_{m, t}\right| \geqq \varepsilon\right\} .
$$

Since $\underline{V}_{m}^{\prime}(t)$ and $\underline{V}_{m}(t)$ are right continuous

$$
\left|\underline{V}_{m}^{\prime}\left(t_{m}^{*}\right)-\underline{V}_{m}\left(t_{m}^{*}\right)\right| \geqq \varepsilon .
$$


Observe that

$$
\begin{aligned}
\left\{\sup _{0 \leqq t \leqq t_{0}+z}\left|\underline{V}_{m, t}^{\prime}-\underline{V}_{m, t}\right| \geqq \varepsilon\right\} & =\left\{t_{m}^{*} \leqq t_{0}+z\right\} \\
& =\left\{t_{m}^{*} \leqq t_{0}+z ;\left|\underline{V}_{m}^{\prime}\left(t^{*}\right)-\underline{V}_{m}\left(t^{*}\right)\right| \geqq \varepsilon\right\}
\end{aligned}
$$

and that

$$
\left|\underline{V}_{m, s}^{\prime}-\underline{V}_{m, s}\right|<\varepsilon, \quad \text { for } \quad s<t_{m}^{*}
$$

By Lemma 3.2

where

$$
\lim _{m \rightarrow 0} \overline{\mathbb{P}}_{m}\left(G_{p}^{m}\right)=1
$$

$$
G_{p}^{m}=\left\{\bar{\omega} \in \bar{\Omega}\left|\sup _{0 \leqq t \leqq t_{0}+z}\right| V_{m, t}^{\prime}(\bar{\omega}) \mid<p c_{m}\right\}, \quad p>0 .
$$

Therefore to establish (5.6) it will suffice to show that

$$
\lim _{m \rightarrow 0} \overline{\mathbb{P}}_{m}\left(\left\{t_{m}^{*} \leqq t_{0}+z,\left|\underline{V}_{m}^{\prime}\left(t_{m}^{*}\right)-\underline{V}_{m}\left(t_{m}^{*}\right)\right| \geqq \varepsilon\right\} \cap G_{p}^{m}\right)=0 .
$$

If we pick $p=1 / 8$ and $\varepsilon<c_{m} / 8$, then on $G_{p}^{m}$

$$
\sup _{t<t_{m}^{*}}\left|\underline{V}_{m, t}\right|<c_{m} / 4
$$

so that with (1.9), if $\left|\underline{v}_{n}\right| \geqq c_{m}$

$$
\left|\underline{v}_{n}^{\prime}\right| \geqq \frac{1-m}{1+m} c_{m}-\frac{2}{1+m}\left|V_{n}\right|>(1 / 4) c_{m}
$$

for $m<1 / 10$. This means that on $G_{p}^{m}$ a fast atom which collides with (Me), bounces off with normal speed $\left|\underline{v}_{n}^{\prime}\right|>c_{m} / 4$ and therefore cannot recollide with the molecule during $\left[0, t_{m}^{*}\right]$.

Remark 5.1. Consequently we have that on $G_{p}^{m}$ within $\left[0, t_{m}^{*}\right]$ the only atoms with which $(\mathrm{Me})$ can recollide are slow atoms, both for the original collision and for recollisions.

This remark, which will be helpful for estimating the effect due to recollisions, follows by noting that (on $G_{p}^{m}$ within $\left[0, t_{m}^{*}\right]$ )

(1) a fast atom cannot recollide with $(\mathrm{Me})$;

(2) an atom which was slow in the first collision will also be slow in recollisions. This may be seen by tracing the paths of $(\mathrm{Me})$ and a colliding fast atom from the collision point backwards in time.

We now compare the velocities of $(\mathrm{Ma})$ and $(\mathrm{Me})$. There are three effects which cause $V_{m}^{\prime}$ to differ from $V_{m}$ :

(1) The change in $\underline{V}_{m}(\omega)$ directly caused by collisions with slow atoms. Let $\mathscr{E}_{m}(t)$ denote the change in $\underline{V}_{m}$ during the time interval $[0, t]$, produced by collisions with slow atoms.

(2) The change in $\underline{V}_{m}^{\prime}(\bar{\omega})$ directly due to the extra-collisions for (Ma) and the change in $\underline{V}_{m}(\bar{\omega})$ due to those fast collisions which don't count in $\underline{V}_{m}^{\prime}$ ("extracollisions" for $(\mathrm{Me}))$. Let $E\left(E^{\prime}\right)$ be an index set for extra-collisions for (Me) ((Ma)) within $\left[t_{0}, t_{m}^{*}\right]$. 
(3) The effect on $\underline{V}^{\prime}$ and the effect on $\underline{V}$ of the fast atoms which collide with (Me) and are also counted in $\underline{V}_{m}^{\prime}$ depend respectively on the velocities of (Ma) and (Me), which may differ. Let $B$ be an index set for these fast atoms which collide within $\left[t_{0}, t_{m}^{*}\right]$.

With $W(i)\left(\underline{W}^{\prime}(i)\right)$ denoting the change in the velocity of $\left.(\mathrm{Me})(\mathrm{Ma})\right)$ due to a collision with a fast atom having index $i$ we have

$$
\begin{aligned}
\left|\underline{V}_{m}^{\prime}\left(t_{m}^{*}\right)-\underline{V}_{m}\left(t_{m}^{*}\right)\right|= & \mid \sum_{i \in B}\left(\underline{W}_{m}^{\prime}(i)-\underline{W}_{m}(i)\right)-\left(\mathscr{E}_{m}\left(t_{m}^{*}\right)-\mathscr{E}_{m}\left(t_{0}\right)\right) \\
& +\sum_{i \in E^{\prime}} \underline{W}_{m}^{\prime}(i)-\sum_{i \in E} \underline{W}_{m}(i)+\underline{V}_{m}^{\prime}\left(t_{0}\right)-\underline{V}_{m}\left(t_{0}\right) \mid \\
\leqq & W_{m}^{(1)}\left(t_{m}^{*}\right)+W_{m}^{(2)}\left(t_{m}^{*}\right)+W_{m}^{(3)}\left(t_{m}^{*}\right)+\left|\underline{V}_{m}^{\prime}\left(t_{0}\right)-\underline{V}_{m}\left(t_{0}\right)\right|
\end{aligned}
$$

where

$$
\begin{aligned}
W_{m}^{(1)}\left(t_{m}^{*}\right) & =\left|\mathscr{E}_{m}\left(t_{m}^{*}\right)-\mathscr{E}_{m}\left(t_{0}\right)\right| \\
W_{m}^{(2)}\left(t_{m}^{*}\right) & =\sum_{i \in E^{\prime}}\left|\underline{W}_{m}^{\prime}(i)\right|+\sum_{i \in E}\left|\underline{W}_{m}(i)\right| \\
W_{m}^{(3)}\left(t_{m}^{*}\right) & =\sum_{i \in B}\left|\underline{W}_{m}^{\prime}(i)-\underline{W}_{m}(i)\right| .
\end{aligned}
$$

Then

$$
\begin{gathered}
\left\{\left|\underline{V}_{m}^{\prime}\left(t_{m}^{*}\right)-\underline{V}_{m}\left(t_{m}^{*}\right)\right| \geqq \varepsilon\right\} \subset \bigcup_{i=1}^{3}\left\{W_{m}^{(i)}\left(t_{m}^{*}\right) \geqq \varepsilon / 4\right\} \\
\cup\left\{\left|\underline{V}_{m}^{\prime}\left(t_{0}\right)-\underline{V}_{m}\left(t_{0}\right)\right| \geqq \varepsilon / 4\right\}
\end{gathered}
$$

and (5.11) follows from (5.5) if

$$
\begin{aligned}
& \lim _{m \rightarrow 0} \overline{\mathbb{P}}_{m}\left(G_{p}^{m} \cap\left\{t_{m}^{*} \leqq t_{0}+z\right\} \cap\left\{W_{m}^{(1)}\left(t_{m}^{*}\right) \geqq \varepsilon / 4\right\}\right)=0, \\
& \lim _{m \rightarrow 0} \overline{\mathbb{P}}_{m}\left(G_{p}^{m} \cap\left\{t_{m}^{*} \leqq t_{0}+z\right\} \cap\left\{W_{m}^{(2)}\left(t_{m}^{*}\right) \geqq \varepsilon / 4\right\}\right)=0, \\
& \lim _{m \rightarrow 0} \overline{\mathbb{P}}_{m}\left(G_{p}^{m} \cap\left\{t_{m}^{*} \leqq t_{0}+z\right\} \cap\left\{W_{m}^{(3)}\left(t_{m}^{*}\right) \geqq \varepsilon / 4\right\}\right)=0,
\end{aligned}
$$

since $\lim _{m \rightarrow 0} \overline{\mathbb{P}}_{m}\left(\left\{\left|V_{m}^{\prime}\left(t_{0}\right)-\underline{V}_{m}\left(t_{0}\right)\right| \geqq \varepsilon / 4\right\}\right)=0$ by hypothesis.

We now establish (5.12)-(5.14) for $p=1 / 8$. All of the estimates in the remainder of Sect. 5 are for $\bar{\omega} \in G_{p}^{m}, p=1 / 8, c_{m} / 8>\varepsilon$, and $m<1 / 10$.

Consider (5.12). We first estimate the total absolute change of the momentum of a slow atom with velocity $\underline{v}(t)$ :

$$
|\Delta \underline{p}|=m\left|\underline{v}\left(t_{m}^{*}\right)-\underline{v}\left(t_{0}\right)\right| ;
$$

we show that

$$
|\Delta \underline{p}| \leqq 7 m c_{m} .
$$

This gives, by conservation of momentum, an upper bound for the total change in the velocity of the molecule produced by one slow atom within $\left[t_{0}, t_{m}^{*}\right]$. We distinguish two cases:

(1) The tangential component $\left|\underline{v}_{t}^{(1)}\right|$ of the velocity $\underline{v}^{(1)}$ of the slow atom in its first collision after $t_{0}$ is $\geqq c_{m}$. 
First observe that the effect of a collision on the velocity $\underline{v}$ of an atom is always to decrease its normal component $v_{n}$. We claim that the same effect is produced between successive collisions, i.e. that

$$
v_{n}^{(i)}(+) \geqq v_{n}^{(i+1)},
$$

where $v_{n}^{(i)}\left(v_{n}^{(i)}(+)\right)$ denotes the normal component of the pre(post)collision velocity of the atom in its $i$-th collision in $\left[t_{0}, t_{m}^{*}\right]$. If this can be shown we easily obtain that

$$
\left|\underline{v}\left(t_{m}^{*}\right)-\underline{v}\left(t_{0}\right)\right| \leqq 4 c_{m},
$$

since if the normal component becomes smaller than $-c_{m} / 4$, further recollisions are impossible: We have that if the atom undergoes $k$ collisions in $\left[t_{0}, t_{m}^{*}\right]$

$$
-c_{m} / 4 \leqq v_{n}^{(k-1)}(+) \leqq v_{n}^{(k-1)} \leqq \ldots \leqq v_{n}^{(1)}(+) \leqq v_{n}^{(1)}<c_{m} .
$$

Therefore

$$
\begin{aligned}
\left|\underline{v}\left(t_{m}^{*}\right)-\underline{v}\left(t_{0}\right)\right| & \leqq \sum_{i=1}^{k} v_{n}^{(i)}(+)-v_{n}^{(i)}\left|\leqq c_{m}+c_{m} / 4+\right| v_{n}^{(k)}(+)-v_{n}^{(k)} \mid \\
& \leqq c_{m}+c_{m} / 4+2 c_{m}+2 c_{m} / 4 \leqq 4 c_{m}
\end{aligned}
$$

where we have used the collision equation (1.9).

To establish the claim (5.16) consider an "equator", on the surface of the molecule, perpendicular to $\underline{v}_{t}^{(1)}$ and passing through the point of the first collision with the atom in $\left[t_{0}, t_{m}^{*}\right]$. Suppose the southern hemisphere is in the direction of $\underline{v}_{t}^{(1)}$. Since $\left|\underline{v}_{t}^{(1)}\right| \geqq c_{m}$ and on $G_{p}^{m}\left|\underline{V}_{m, t}\right| \leqq c_{m} / 4$ for $t<t_{m}^{*}$ the collision point in subsequent recollisions will always be in the southern hemisphere and the speed of the atom will remain at least as large as $c_{m}$. Now consider a plane perpendicular to the velocity $v^{(i)}(+)$ of the atom after the $i$-th collision passing through the point of the $i$-th collision and fixed in (i.e. moving with) the molecule. Since $\left|\underline{v}^{(i)}(+)\right| \geqq c_{m}$ and $|\underline{V}| \leqq c_{m} / 4$ the next collision (if there is one) with the atom must occur on the side of the plane in the direction of $\underline{v}^{(i)}(+)$, i.e. (5.16) holds.

$$
\text { (2) }\left|\underline{v}_{t}^{(1)}\right|<c_{m} \text {. }
$$

If the tangential component grows bigger than $c_{m}$ at some time $t_{1} \leqq t_{m}^{*}$, we can apply (1): A little thought shows that $\left|\underline{v}\left(t_{1}\right)\right| \leqq \sqrt{2} c_{m}$ and we obtain with $\left|\underline{v}\left(t_{0}\right)\right| \leqq \overline{2} c_{m}$

$$
\left|\underline{v}\left(t_{m}^{*}\right)-\underline{v}\left(t_{0}\right)\right|=\left|\underline{v}\left(t_{m}^{*}\right)-\underline{v}\left(t_{1}\right)\right|+\left|\underline{v}\left(t_{1}\right)-\underline{v}\left(t_{0}\right)\right|=4 c_{m}+2 \sqrt{2} c_{m} .
$$

If the tangential component is always less than $c_{m},\left|\underline{v}\left(t_{m}^{*}\right)\right| \leqq 2 c_{m}$, since the absolute value of the normal component cannot become bigger than $c_{m}+c_{m} / 2$. Then

$$
\left|\underline{v}\left(t_{m}^{*}\right)-\underline{v}\left(t_{0}\right)\right| \leqq(2 \sqrt{2}) c_{m} ;
$$

this combined with (1) proves (5.15).

To estimate the total effect $W_{m}^{(1)}\left(t_{m}^{*}\right)$ of the slow atoms, we have to multiply (5.15) by the number $N\left(t_{m}^{*}\right)$ of distinct slow atoms which hit $(\mathrm{Me})$ in $\left[t_{0}, t_{m}^{*}\right]$. But in 
view of Remark $5.1 N\left(t_{m}^{*}\right) \leqq N_{m}=$ the number of first collisions with slow atoms within $[0, T]$ in which $(\mathrm{Me})$ has a speed $V \leqq c_{m}$.

$N_{m}$ can be estimated using the rates (3.4), since for the rate of first collisions of slow atoms with the molecule Lemma 3.1 provides an upper bound. Setting in (3.4) $\left(v_{n}-V_{n}\right)_{+}=2 c_{m}$ we obtain with $E(\cdot)$ as expectation $\left(\lambda=r^{2}=1\right)$

$$
E\left(N_{m}\right) \leqq 8 \pi c_{m} \operatorname{Tm}^{-1 / 2},
$$

and we obtain using (5.15)

$$
\begin{gathered}
\overline{\mathbb{P}}_{m}\left(G_{p}^{m} \cap\left\{t_{m}^{*} \leqq t_{0}+z\right\} \cap\left\{W_{m}^{(1)}\left(t_{m}^{*}\right) \geqq \varepsilon / 4\right\}\right) \leqq \mathbb{P}_{m}\left(\left\{N_{m}|\Delta p| \geqq \varepsilon / 4\right\}\right) \\
\quad=\mathbb{P}_{m}\left(\left\{N_{m} \geqq \varepsilon /\left(28 m c_{m}\right)\right\}\right) \leqq 28 m c_{m} E\left(N_{m}\right) / \varepsilon \sim c_{m}^{2} m^{1 / 2} T / \varepsilon .
\end{gathered}
$$

Since $c_{m}=m^{-1 / 5}$ the r.h.s. of the last equation goes to zero with $m$.

Next we establish (5.13).

For the rate of the occurrence of extra collisions, including "extra collisions" for $(\mathrm{Me})$, we have

$$
\tilde{R}_{m}\left(\underline{V}^{\prime}, \underline{V}, \underline{v}_{n}\right)=\left|r_{m}\left(\underline{V}^{\prime}, \underline{v}_{n}\right)-r\left(\underline{V}, \underline{v}_{n}\right)\right| .
$$

Since $\sup _{t<t_{m}^{*}}\left|\underline{V}_{m, t}^{\prime}\right|<c_{m} / 8, \sup _{t<t_{m}^{*}}\left|\underline{V}_{m, t}\right|<c_{m} / 4$ and $\left|\underline{v}_{n}\right| \geqq c_{m}$ for the extra collisions, we obtain using (5.1) and (3.4)

$$
\tilde{R}_{m}\left(\underline{V}^{\prime}, \underline{V}, \underline{v}_{n}\right)=m^{-1 / 2}\left|\underline{V}_{n}^{\prime}-\underline{V}_{n}\right| f_{m}^{1}\left(v_{n}\right) .
$$

In view of (5.10) $\left|\underline{V}_{n}^{\prime}-\underline{V}_{n}\right| \leqq \varepsilon$ (for $t<t_{m}^{*}$ ); therefore we consider the Poisson field $Y_{m}$ on the $t-\underline{v}_{n}$ space determined by the rates

$$
R_{m}^{P}\left(\varepsilon, \underline{v}_{n}\right)=m^{-1 / 2} \varepsilon f_{m}^{1}\left(v_{n}\right)
$$

which majorizes the rate $\tilde{R}_{m}\left(\underline{V}^{\prime}, \underline{V}, \underline{v}_{m}\right)$ of the actual process of extra collisions. Hence the correlation functions for the extra collisions in the actual process within $\left[t_{0}, t_{m}^{*}\right]$ are less than those of the process $Y_{m}$. This may be seen by introducing a new "independent" process $\tilde{Y}_{m}$ defined by the rates

$$
R_{m}^{\prime}\left(\underline{V}^{\prime}, \underline{V}, \underline{v}_{n}\right)=R_{m}^{P}\left(\varepsilon, \underline{v}_{n}\right)-\tilde{R}_{m}\left(\underline{V}^{\prime}, \underline{V}, \underline{v}_{n}\right)
$$

so that $Y_{m}$ arises by "combining" $\tilde{Y}_{m}$ with the actual process of extra collisions. For the change $\Delta \underline{V}_{m}$ of the velocity of the molecule due to a fast atom we have according to the collision equation $(1.10)\left(V \leqq c_{m}\right)$

$$
\left|\Delta \underline{V}_{m}\right| \leqq \frac{2 m}{1+m}\left(\left|\underline{v}_{n}\right|+c_{m}\right) \leqq \frac{4 m}{1+m}\left|\underline{v}_{n}\right| .
$$

Using $Y_{m}$ we obtain for (5.13) the inequality

$$
\begin{aligned}
\overline{\mathbb{P}}_{m}\left(G_{p}^{m}\right. & \left.\cap\left\{t_{m}^{*} \leqq t_{0}+z\right\} \cap\left\{W_{m}^{(2)}\left(t_{m}^{*}\right) \geqq \varepsilon / 4\right\}\right) \\
\leqq & \operatorname{Prob}\left(\sum_{i=1}^{N} \frac{4 m}{1+m} v_{n}^{(i)} \geqq \varepsilon / 4\right),
\end{aligned}
$$


where $\left(\underline{v}_{n}^{(1)}, \ldots, \underline{v}_{n}^{(N)}\right)$ represents the "extra collisions" in $\left[t_{0}, t_{0}+z\right]$ arising from the Poisson field $Y_{m}$ and $v_{n}^{(i)}=\left|\underline{v}_{n}^{(i)}\right|$. With $E_{m}(\cdot)$ denoting the expectation corresponding to $Y_{m}$ we obtain using (5.17)

$$
\begin{aligned}
E_{m}\left(\sum_{i=1}^{N} \frac{4 m}{1+m} v_{n}^{(i)}\right) & =(4 m / 1+m) E_{m}\left(\sum_{i=1}^{N} v_{n}^{(i)}\right) \\
& =(4 m / 1+m) 4 \pi \varepsilon z m^{-1} \int_{c_{m} \sqrt{m}}^{\infty} v f^{1}(v) d v \leqq 16 \pi \varepsilon z \Phi_{1} \leqq \varepsilon / 8
\end{aligned}
$$

for our choice of $z=\left(128 \pi \Phi_{1}\right)^{-1}$. Hence the r.h.s. of (5.18) can be estimated as follows: With

$$
\begin{gathered}
J:=\sum_{i=1}^{N} v_{n}^{(i)}, \\
\operatorname{Prob}\left(\frac{4 m}{1+m} J \geqq \varepsilon / 4\right) \leqq \operatorname{Prob}\left(J \geqq 2 E_{m}(J)\right)=\operatorname{Prob}\left(J-E_{m}(J) \geqq E_{m}(J)\right) \\
\leqq \frac{E_{m}\left(\left[J-E_{m}(J)\right]^{2}\right)}{E_{m}(J)^{2}}
\end{gathered}
$$

by Chebychev's inequality. Using (5.19) and the independence of the "extra collisions" in the process $Y_{m}$ we obtain with

$$
\frac{\int_{c_{m}}^{\infty} v_{n}^{k} f_{m}^{1}\left(v_{n}\right) d v_{n}}{\int_{c_{m}}^{\infty} f_{m}^{1}\left(v_{n}\right) d v_{n}}=\left\langle v_{n}^{k}\right\rangle, \quad k=1,2
$$

and some basic properties of Poisson random fields

$$
\begin{aligned}
E_{m}(J) & =\left\langle v_{n}\right\rangle E_{m}(N) \\
E_{m}\left(J^{2}\right) & =E_{m}(N)\left\langle v_{n}^{2}\right\rangle+E_{m}(N(N-1))\left\langle v_{n}\right\rangle^{2}=E_{m}(N)\left\langle v_{n}^{2}\right\rangle+E_{m}(N)^{2}\left\langle v_{n}\right\rangle^{2} .
\end{aligned}
$$

For the r.h.s. of (5.20) we thus obtain $E_{m}(N)^{-1}\left\langle v_{n}^{2}\right\rangle /\left\langle v_{n}\right\rangle^{2} \underset{m \rightarrow 0}{\longrightarrow} 0$, since $\left\langle v_{n}^{2}\right\rangle \sim m^{-1},\left\langle v_{n}\right\rangle \sim m^{-1 / 2}$ and $E_{m}(N) \sim m^{-1 / 2}$.

In view of (5.20) and (5.18), (5.13) follows [in case $\left\langle v_{n}\right\rangle=0,(5.13)$ is trivial].

We are left with (5.14), and because of the coupling we have chosen this is an easy estimate. With $\underline{W}_{i}\left(\underline{W}_{i}^{\prime}\right)$ as the effect of atom $i$, which collides with $(\mathrm{Me})((\mathrm{Ma}))$ at time $s \leqq t_{m}^{*}$ with normal velocity $\underline{v}_{n}^{(i)}$, we have

$$
\begin{aligned}
\left|\underline{W}_{i}^{\prime}-\underline{W}_{i}\right| & =\mid \underline{V}_{n}^{\prime}(s-)-\left(\frac{1-m}{1+m} \underline{V}_{n}^{\prime}(s-)+\frac{2 m}{1+m} \underline{v}_{n}^{(i)}\right) \\
& -\left(\underline{V}_{n}(s-)-\left(\frac{1-m}{1+m} \underline{V}_{n}(s-)+\frac{2 m}{1+m} \underline{v}_{n}^{(i)}\right)\right) \mid \\
& =\frac{2 m}{1+m}\left|\underline{V}_{n}^{\prime}(s-)-\underline{V}_{n}(s-)\right| \leqq 2 m \varepsilon /(1+m)
\end{aligned}
$$

using (5.10). 
Hence

$$
W_{m}^{(3)}\left(t_{m}^{*}\right) \leqq \frac{2 m}{1+m} \varepsilon N_{m}(z),
$$

where $N_{m}(z)$ is the number of collisions involving (Ma) within $\left[t_{0}, t_{0}+z\right]$. Since the total rate of collisions for the Markov process is $\bar{\lambda}_{m}$, we obtain

$$
E\left(N_{m}(z)\right)=: \bar{N}_{m, z}=\bar{\lambda}_{m} z \leqq 4 \pi z m^{-1} \Phi_{1} .
$$

We therefore obtain

$$
\begin{aligned}
& \overline{\mathbb{P}}_{m}\left(G_{p}^{m} \cap\left\{t_{m}^{*} \leqq t_{0}+z\right\} \cap\left\{W_{m}^{(3)}\left(t_{m}^{*}\right) \geqq \varepsilon / 4\right\}\right) \\
& \quad \leqq \overline{\mathbb{P}}_{m}\left(\left\{\frac{2 m}{1+m} \varepsilon N_{m}(z) \geqq \varepsilon / 4\right\}\right)=\overline{\mathbb{P}}_{m}\left(\left\{N_{m}(z) \geqq 1+m / 8 m\right\}\right) \\
& \quad \leqq \overline{\mathbb{P}}_{m}\left(\left\{N_{m}(z)>2 \bar{N}_{m, z}\right\}\right)=\overline{\mathbb{P}}_{m}\left(\left\{N_{m}(z)-\bar{N}_{m, z}>\bar{N}_{m, z}\right\}\right) \\
& \quad \leqq E\left(\left(N_{m}(z)-\bar{N}_{m, z}\right)^{2}\right) / \bar{N}_{m, z}^{2}=\bar{N}_{m, z} / \bar{N}_{m, z}^{2}=\bar{N}_{m, z}^{-1} \rightarrow 0,
\end{aligned}
$$

by (5.21), using the fact that $z$ is such that $(1+m) / 8 m>2 \bar{N}_{m, z}$. This completes the proof of Lemma 5.2.

\section{General Velocity Distribution}

In this section we extend Theorem 2.1 to a general, i.e. not necessarily spherical symmetric, velocity distribution $f(\underline{v})$. Whenever possible we use the same notation as in the previous sections. Clearly the description of the mechanical process $\underline{V}_{m}$ is the same as before. Moreover, what has been done in the previous sections can easily be adapted to this slightly more general setting. In particular, the treatment in Sect. 5 of the closeness of the paths applies without essential modification. We now describe the major differences.

Throughout Sects. 3-5, $f_{m}^{1}$ should now be replaced by $f_{m}^{e_{n}}$, the distribution of $\underline{e}_{n} \cdot \underline{v}$. Note that $N_{m}(\underline{V})$ [Eq. (3.6)] now need not equal $N_{m}(0)$, even for $|\underline{V}| \leqq c_{m}$. Thus, defining $\bar{\lambda}_{m}=\bar{\lambda}_{m}(\underline{V})$ by

$$
\bar{\lambda}_{m}=\lambda_{m} r^{2} \min \left[N_{m}(\underline{V}), \sup _{\left|\underline{V}^{\prime}\right| \leqq c_{m}} N_{m}\left(\underline{V}^{\prime}\right)\right]
$$

in place of (3.8), the Markov approximation $\tilde{V}_{m}$ (defined exactly as in Definition 3.1) will now have a process dependent mean waiting time $\bar{\lambda}_{m}^{-1}$.

Integrals of the form $\int_{\alpha}^{\infty} y^{k} f_{m}^{1}(y) d y$, appearing in Sect. 5, which are not already embedded in an $\Omega$-integration should be replaced by

$$
\begin{aligned}
(4 \pi)^{-1} \int d \Omega \int_{\alpha}^{\infty} y^{k} f_{m}^{\underline{e}_{n}}(y) d y= & (4 \pi)^{-1} \int d \Omega \int_{\underline{e}_{n} \cdot \underline{v} \geqq \alpha}\left(\underline{e}_{n} \cdot \underline{v}\right)^{k} f_{m}(\underline{v}) d \underline{v} \\
& \left(\leqq \int|\underline{v}|^{k} f_{m}(\underline{v}) d \underline{v}=m^{-k / 2} \int|\underline{\mid}|^{k} f(\underline{v}) d \underline{v}\right) .
\end{aligned}
$$

Definition 6.1. Let $\mathscr{A}, \mathscr{D}$ be real $3 \times 3$ matrices, symmetric and positive semidefinite. The (generalized) Ornstein-Uhlenbeck process $\underline{V}_{0}$ is the diffusion process with generator $A$ given by

$$
A \uparrow_{C_{c}^{\infty}}=-\underline{V} \cdot \mathscr{A} \cdot \nabla+\frac{1}{2} \nabla \cdot \mathscr{D} \cdot \nabla .
$$


We now give the extension of Theorem 2.1:

Theorem 6.1. Let $f(\underline{v})$ be any probability density with $\int v^{4} f(\underline{v}) d \underline{v}<\infty$ and

$$
\int|\underline{v}| \underline{v} f(\underline{v}) d \underline{v}=0 .
$$

Then

$$
\underline{V}_{m} \stackrel{\vartheta}{\Rightarrow} \underline{V}_{0}
$$

where $\underline{V}_{0}$ is the Ornstein-Uhlenbeck process with drift and diffusion coefficients ( $\mathbb{1}$ $=$ unit matrix and $\underline{v} \underline{v}=$ tensor product)

$$
\begin{aligned}
& \mathscr{A}=M^{-1} \lambda r^{2} \pi \int|\underline{v}| f(\underline{v})\left(\mathbb{1}+\frac{\underline{v} \underline{v}}{|\underline{v}|^{2}}\right) d \underline{v}, \\
& \mathscr{D}=M^{-2} \lambda r^{2} \frac{\pi}{3} \int|\underline{v}|^{3} f(\underline{v})\left(\mathbb{1}+3 \frac{\underline{v} \underline{v}}{|\underline{v}|^{2}}\right) d \underline{v} .
\end{aligned}
$$

Remark. The condition (6.2) is needed for the existence of the limit $\lim _{m \rightarrow 0} \tilde{A}^{(m)}$ (recall that $\tilde{A}^{(m)}$ is the generator of $\tilde{V}_{m}$ ); the 1.h.s. of (6.2) may be interpreted as the net force acting on the molecule due to collisions with the atoms. We will see below that without (6.2) the drift becomes infinite. As a side remark let us mention that (6.2) can always be fulfilled by choosing an appropriate coordinate system, i.e. if (6.2) does not hold one can find an $\underline{a}$ such that for $\tilde{f}(\underline{v})=f(\underline{v}-\underline{a})(6.2)$ is fulfilled. [The scaling should then be applied to $\tilde{f}(\underline{v})$, i.e. $\tilde{f}_{m}(\underline{v})=m^{3 / 2} \tilde{f}\left(m^{1 / 2} \underline{v}\right)=m^{3 / 2} f\left(m^{1 / 2} \underline{v}-\underline{a}\right)$.] Proof of Theorem 6.1. All that remains is to establish

Lemma 6.1. $\underline{V}_{m} \stackrel{\vartheta}{\Rightarrow} \underline{V}_{0}$.

Proof. We establish the convergence of the generator as in Sect. 4. We note first that the generator $\tilde{A}^{(m)}$ of $\tilde{V}_{m}$ is given by

$$
\tilde{A}^{(m)} h\left(\underline{V}^{0}\right)=\bar{\lambda}_{m}\left(\underline{V}^{0}\right)\left(-h\left(\underline{V}^{0}\right)+\int G\left(\underline{V}^{0}, d \underline{V}\right) h(\underline{V})\right)
$$

on the domain $\Delta \tilde{A}^{(m)}=C_{0}$.

This follows from the observation that

$$
\begin{aligned}
\tilde{E}_{\underline{V}^{0}}^{(m)}\left(h\left(\tilde{V}_{m, t}\right)\right)= & e^{-\bar{\lambda}_{m}\left(\underline{V}^{0}\right) t} h\left(\underline{V}^{0}\right)+\bar{\lambda}_{m}\left(\underline{V}^{0}\right) \int_{0}^{t} d t^{\prime} \\
& \cdot \int\left(e^{-\bar{\lambda}_{m}\left(\underline{V}^{0}\right) t^{\prime}} G_{m}\left(\underline{V}^{0}, d \underline{V}\right) e^{-\bar{\lambda}_{m}(\underline{V})\left(t-t^{\prime}\right)} h(\underline{V})\right)+O\left(t^{2}\right) \\
= & \left(1-\bar{\lambda}_{m}\left(\underline{V}^{0}\right) t\right) h\left(\underline{V}^{0}\right)+\bar{\lambda}_{m}\left(\underline{V}^{0}\right) t \int G_{m}\left(\underline{V}^{0}, d \underline{V}\right) h(\underline{V})+O\left(t^{2}\right) .
\end{aligned}
$$

We write the integrals $J_{i}$ [Eq. (4.7) with $f^{e_{n}}$ replacing $f^{1}$ ] in the form

$$
J_{i}=\left(\bar{\lambda}_{m} q_{m}^{i-1} / N_{m}\left(\underline{V}^{0}\right)\right) \int d \Omega \int_{\underline{e}_{n} \cdot \underline{v} \geqq c_{m}}\left(\left(\underline{e}_{n} \cdot\left(\underline{v}-\underline{V}^{0}\right)\right)_{+}\right)^{i} \frac{\left(\underline{e}_{n} \cdot \nabla\right)^{i-1}}{(i-1) !} h\left(\underline{V}_{i}\right) f_{m}(\underline{v}) d \underline{v} .
$$

We assume $h(\underline{x})=0$ for $|\underline{x}|>b$ and we choose $m$ so small that $c_{m}>b$. Using the fact that

$$
\int|\underline{v}|^{4} f(\underline{v}) d \underline{v}<\infty,
$$

we obtain, as in Sect. 4 , that $J_{4} \underset{m \rightarrow 0}{\longrightarrow} 0$, uniformly in $\underline{V}^{0}$. 
For $J_{3}$ we have

$$
\begin{aligned}
J_{3}= & \frac{1}{2} \bar{\lambda}_{m} q_{m}^{2} r^{2} \nabla \cdot\left(\int d \underline{v} f_{m}(\underline{v}) \int_{e_{n} \cdot v \geqq c_{m}} d \Omega\left(\underline{e}_{n} \cdot \underline{v}\right)^{3} \underline{e}_{n} \underline{e}_{n}\right) \\
& \cdot \nabla h\left(\underline{V}^{0}\right)+O\left(m^{1 / 2}\right) \underset{m \rightarrow 0}{\longrightarrow} \frac{2 \lambda r^{2}}{M^{2}} \nabla \\
& \cdot\left(\int d \underline{v} f(\underline{v}) \int_{\underline{e}_{n} \cdot \underline{v} \geqq 0} d \Omega\left(\underline{e}_{n} \cdot \underline{v}\right)^{3} \underline{e}_{n} \underline{e}_{n}\right) \cdot \nabla h\left(\underline{V}^{0}\right) \\
= & \frac{1}{2} \nabla \cdot \mathscr{D} \cdot \nabla h\left(\underline{V}^{0}\right),
\end{aligned}
$$

uniformly in $\underline{V}^{0}$, using $(k=0,1,2,3, \ldots)$

$$
\int_{\underline{e}_{n} \cdot \underline{v} \geqq 0} d \Omega\left(\underline{e}_{n} \cdot \underline{v}\right)^{k} \underline{e}_{n} \underline{e}_{n}=\frac{2 \pi}{(k+1)(k+3)}|\underline{v}|^{k}\left(\mathbb{1}+k \frac{\underline{v} \underline{v}}{|\underline{v}|^{2}}\right) .
$$

For $J_{2}$ we obtain, again using (6.4),

$$
\begin{aligned}
J_{2}= & q_{m} \lambda_{m} r^{2}\left[\int d \underline{v} f_{m}(\underline{v})\left(\underline{v}-2 \underline{V}^{0}\right) \cdot\left(\int_{\underline{e}_{n} \cdot \underline{v} \geqq c_{m}} d \Omega\left(\underline{e}_{n} \cdot \underline{v}_{)} \underline{e}_{n} \underline{e}_{n}\right) \cdot \nabla h\left(\underline{V}^{0}\right)\right]+O\left(m^{1 / 2}\right)\right. \\
= & q_{m} \lambda_{m} r^{2}\left[\int d \underline{v} f_{m}(\underline{v})\left(\underline{v}-2 \underline{V}^{0}\right) \cdot\left(\int_{\underline{e}_{n} \cdot \underline{v} \geqq 0} d \Omega\left(\underline{e}_{n} \cdot \underline{v}\right) \underline{e}_{n} \underline{e}_{n}\right) \cdot \nabla h\left(\underline{V}^{0}\right)\right]+O\left(m^{1 / 10}\right) \\
= & q_{m} \lambda_{m} r^{2} m^{-1} \frac{1}{2} \pi \int d \underline{v} f(\underline{v})\left(\underline{v} \mid \underline{v} \cdot \nabla h\left(\underline{V}^{0}\right)-q_{m} \lambda_{m} r^{2} m^{1 / 2} \frac{1}{2} \pi \underline{V}^{0}\right. \\
& \cdot \int d \underline{v} f(\underline{v}) v\left(\mathbb{1}+\frac{\underline{v} \underline{v}}{|\underline{v}|^{2}}\right) \cdot \nabla h\left(\underline{V}^{0}\right)+O\left(m^{1 / 10}\right) \underset{m \rightarrow 0}{\longrightarrow}-\underline{V}^{0} \cdot \mathscr{A} \cdot \nabla h\left(\underline{V}^{0}\right),
\end{aligned}
$$

uniformly in $\underline{V}^{0}$, using (6.2).

We conclude the section with two remarks:

(1) It is not necessary to assume that the radius of the molecule is fixed. All that our argument requires is that $\lambda r^{2} \sim m^{-1 / 2}$, so that by appropriately scaling $\lambda$ with $m$, the radius of the molecule can be scaled as well.

(2) The absolute continuity of the velocity distribution is not essential. It is used (in the Appendix) only to preclude multiple collisions, which render the definition of a natural deterministic time evolution for the mechanical system problematical. However, if we do not insist on a deterministic evolution, the motion can easily be continued past multiple collisions: randomly choose the order in which the collisions are to occur. Everything which we have done in this paper then applies essentially unchanged.

\section{Appendix}

The mechanical process $\underline{V}_{m}$ has been defined only for good configurations of the Poisson field, describing the atoms. These give rise, in any finite time interval, to at most finitely many collisions none of which are multiple collisions. We denote the set of such configurations by $\hat{\Omega}\left(\hat{\Omega}\right.$ of course depends upon $\underline{X}^{0}$ and $\left.\underline{V}^{0}\right)$.

Proposition A.1. $\mathbb{P}_{m}(\hat{\Omega})=1$.

Proof. We first consider systems (consisting of a "molecule" undergoing elastic collisions with "atoms") in which the number of atoms is finite $(n)$, which we call finite systems. 
Lemma A.1. For finite systems at most finitely many collisions can occur (in any time interval).

This is a special case of a result of Vaserstein [12], concerning the number of collisions in systems with repulsive interactions.

Lemma A.2. For any initial position $\underline{X}^{0}$ and velocity $\underline{V}^{0}$ of the molecule in a finite system, the Lebesgue measure of the set of bath configurations giving rise to multiple collisions is zero.

Proof. Label the atoms by $i=1, \ldots, n$. We first show that

$$
\mu_{n+1}(\mathscr{M})=0 \text {, }
$$

where $\mathscr{M} \subset \mathbb{R}^{6(n+1)}$ is the set of phase points for which a multiple collision occurs, and for any $N, \mu_{N}$ denotes the $6 N$ dimensional Lebesgue measure. Clearly $\mathscr{M} \subset \bigcup_{i=1}^{n} \mathscr{M}_{i}$, where $\mathscr{M}_{i} \subset \mathbb{R}^{6(n+1)}$ is the subset of $\mathscr{M}$ for which the first multiple collision involves atom $i$.

For any $t$, consider the set $\mathscr{M}_{i}(t)$ of phase points in $\mathscr{M}_{i}$ for which the first multiple collision is also the first collision involving atom $i$ after time $t$. Let $t_{k}$ be an enumeration of the positive rationals. Then

$$
\mathscr{M}_{i} \subset \bigcup_{k} \mathscr{M}_{i}\left(t_{k}\right)
$$

and to establish (A.1) it suffices to show that

for all $t$.

$$
\mu_{n+1}\left(\mathscr{M}_{i}(t)\right)=0
$$

Consider the section

$$
\mathscr{M}_{i}^{\xi}=\left\{\underline{q}_{i} \in \mathbb{R}^{3} \mid\left(q_{i}, \xi\right) \in \mathscr{M}_{i}(0)\right\},
$$

where $\xi \in \mathbb{R}^{6(n+1)-3}$ represents all the coordinates except the position $\underline{q}_{i}$ of atom $i$. The path of the molecule until either a multiple collision or a collision with atom $i$ occurs is completely determined by $\xi$. Since by Lemma A.1 only finitely many collisions can occur, it follows that for fixed $\xi$ the first collision involving atom $i$ can occur as the first multiple collision for only finitely many times $t^{(k)}$ and positions $\underline{X}^{(k)}$ of the molecule. Since $\underline{v}_{i}$ is fixed, it follows that $\mathscr{M}_{i}^{\xi}$ is contained in a finite union of hypersurfaces (spheres). Thus $\mathscr{M}_{i}^{\xi}$ has vanishing Lebesgue measure, and $\mu_{n+1}\left(\mathscr{M}_{i}(0)\right)=0$.

Let $\varphi_{t}$ be the evolution mapping, from time 0 to time $t$, arising from the dynamics of elastic collisions. On $M_{i}(t) \varphi_{t}$ is well defined and preserves Lebesgue measure. Moreover, $\varphi_{t}\left(\mathscr{M}_{i}(t)\right) \subset \mathscr{M}_{i}(0) ;($ A.2), and hence (A.1), follow.

The lemma asserts that

$$
\mu_{n}\left(\mathscr{M}\left(\underline{X}^{0}, \underline{V}^{0}\right)\right)=0
$$

for all $\underline{X}^{0}, \underline{V}^{0} \in \mathbb{R}^{3}$, where

$$
\mathscr{M}\left(\underline{X}^{0}, \underline{V}^{0}\right)=\left\{\left(\underline{q}_{1}, \underline{v}_{1}, \ldots, \underline{q}_{n}, \underline{v}_{n}\right) \mid\left(\underline{X}^{0}, \underline{V}^{0}, \underline{q}_{1}, \underline{v}_{1}, \ldots, \underline{q}_{n}, \underline{v}_{n}\right) \in \mathscr{M}\right\} .
$$

It follows from (A.1) that (A.3) holds for almost all $\underline{X}^{0}, \underline{V}^{0}$. Since the sets $\mathscr{M}\left(\underline{X}^{0}, \underline{V}^{0}\right)$ for different values of $\underline{X}^{0}, \underline{V}^{0}$ are related by translations, the lemma follows. 
We now turn to Proposition A.1. Without loss of generality we may assume that $\lambda=1, m=1$, and $\underline{X}^{0}=0$, and we write $\mathbb{P}$ for $\mathbb{P}_{1}$. Since, at least initially, the number of atoms in a finite volume is (almost surely) finite, our concern is with atoms coming from far away. The key ingredient is establishing the fact that atoms far away from the molecule are well located in the sense that they cannot come close to the molecule in a finite amount of time.

Consider the set $A_{k} \subset \Omega(k \in \mathbb{N})$ of configurations for which there is at least one atom $(\underline{q}, \underline{v})$ with $(\varepsilon>0$ is fixed)

We first show that

$$
k<|\underline{q}| \leqq k+1 \text { and }|\underline{v}| \geqq \varepsilon k .
$$

$$
\mathbb{P}\left(\lim \sup A_{k}\right)=0 \text {. }
$$

According to the Borel-Cantelli Lemma (A.5) holds if $\sum_{k} \mathbb{P}\left(A_{k}\right)<\infty$. Let $N_{k}$ denote the number of atoms satisfying (A.4) (at time 0). Then

$$
\begin{aligned}
\mathbb{P}\left(A_{k}\right) & =\mathbb{P}\left(N_{k} \geqq 1\right) \leqq E\left(N_{k}\right)=\int_{k \leqq q \leqq k+1} \int_{|v| \geqq \varepsilon k} f(\underline{v}) d \underline{v} d \underline{q} \\
& =4 \pi\left(k^{2}+k+1 / 3\right) \int_{|v| \geqq \varepsilon k} f(\underline{v}) d \underline{v},
\end{aligned}
$$

where we have used (1.1). But $\int_{|\underline{v}| \geqq \varepsilon k} f(\underline{v}) d \underline{v} \leqq(\varepsilon k)^{-4} \int|\underline{v}|^{4} f(\underline{v}) d \underline{v} \sim k^{-4}$, since the velocity distribution has a finite fourth moment. Thus $\sum_{k} \mathbb{P}\left(A_{k}\right)<\infty$, and we have (A.5).

Let $\tau=\sup \{s \geqq 0$ : the evolution $X(t)$ of the molecule is well defined for all $0 \leqq t \leqq s\}$, i.e., $\tau$ is the time at which the evolution ceases to be well defined. Proposition A. 1 is equivalent to the statement

$$
\tau=\infty, \text { almost surely. }
$$

For any $T>0$, and integers $k>j>0$, let $A_{k j}^{T} \subset \Omega$ denote the event that no atom outside the sphere $|q|=k$ is transported by free motion into $|q| \leqq j$ by time $T$.

It follows from (A.5) that for any $j$ and any $T>0$

$$
\mathbb{P}\left(\bigcup_{k>j} A_{k j}^{T}\right)=1
$$

Note that it easily follows from (A.7) that $\tau>0$ almost surely. To use (A.7) effectively for times greater than 0 we must show that the molecule cannot move too far.

For $T>0$, let $\tau_{T}=\tau \wedge T$ and let $B_{j}^{T} \subset \Omega$ be the event that the molecule leaves the ball $|q| \leqq j$ before time $\tau_{T}$. In order for $B_{j}^{T}$ to occur, the molecule must attain a speed $V>\bar{j}=j / T$, i.e. an energy $U>\frac{1}{2} M \bar{j}^{2}=\bar{U}$, by time $\tau_{T}$. Therefore

$$
B_{j}^{T} \subset\left\{\sup _{0 \leqq t<\tau_{T}} U(t)>\bar{U}\right\}=\left\{t_{T}^{*}<\tau_{T}\right\}
$$

where

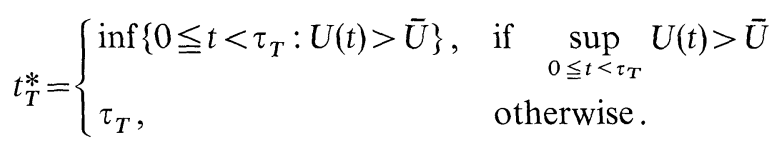


Thus

$$
\mathbb{P}\left(B_{j}^{T}\right) \leqq \mathbb{P}\left\{t_{T}^{*}<\tau_{T}, U\left(t_{T}^{*}\right) \geqq \bar{U}\right\},
$$

since $\left\{t_{T}^{*}<\tau_{T}\right\} \subset\left\{U\left(t_{T}^{*}\right) \geqq \bar{U}\right\}$.

Observe that for the change $\Delta U$ in the energy of the molecule due to all collisions with a single atom whose initial speed is $v$, we have

$$
\Delta U \leqq v^{2} / 2 \text {. }
$$

Let $\underline{v}_{1}, \ldots, \underline{v}_{N}$ denote the initial $(t=0)$ velocities of atoms involved by time $t_{T}^{*}$ in collisions in which the molecule has (precollision) speed $V \leqq \bar{j}$. We obtain from (A.8) that

$$
\mathbb{P}\left\{t_{T}^{*}<\tau_{T} ; U\left(t_{T}^{*}\right) \geqq \bar{U}\right\} \leqq \mathbb{P}\left\{\sum_{i=1}^{N}\left|\underline{v}_{i}^{2}\right| \geqq M \bar{j}^{2}-2 U(0)\right\} \leqq \frac{E\left(\sum_{i=1}^{N}\left|\underline{v}_{i}\right|^{2}\right)}{M \bar{j}^{2}-2 U(0)} .
$$

Using the rates (3.4) we obtain

$$
E\left(\sum_{i=1}^{N}\left|v_{i}\right|^{2}\right) \leqq 4 \pi T \int(|\underline{v}|+\bar{j})|\underline{v}|^{2} f(\underline{v}) d \underline{v}=O(j),
$$

since $f(\underline{v})$ has a finite third moment. Therefore, for all $T>0$

$$
\lim _{j \rightarrow \infty} \mathbb{P}\left(B_{j}^{T}\right)=0 \text {. }
$$

It easily follows from (A.7), (A.9), and Lemma A.1 that if $\tau<\infty$, then, almost surely, the molecule suffers at most finitely many collisions before time $\tau$ and the evolution ceases to be well defined at $\tau$ only because a multiple collision occurs at $\tau$. Since the velocity distribution of the atoms in the Poisson field is absolutely continuous, it is not difficult to see that by Lemma A.2 we must therefore have $\tau=\infty$ almost surely.

\section{References}

1. Nelson, E.: Dynamical theories of brownian motion. Princeton: Princeton University Press 1967

2. Holley, R.: Z. Wahrsch. Verw. Gebiete 17, 181-219 (1971)

3. Hennion, H. : Z. Wahrsch. Verw. Gebiete 25, 123-154 (1973)

4. Brunnschweiler, A.: Thesis, Ecole Polytechnique Federale Lausanne, 1976

5. Renyi, A.: Stud. Sci. Math. Hung. 2, 119-123 (1967)

6. Billingsley, P.: Convergence of probability measures. New York: John Wiley and Sons 1968

7. Dynkin, E.B.: Markov processes. I, II. Berlin, Göttingen, Heidelberg: Springer 1965

8. Kurtz, Th. : Ann. Probab. 4, 618-642 (1975)

9. Skorohod, A.V.: Theory Probab. Its Appl. (USSR) 3, 202-246 (1957)

10. Nelson, E.: Topics in dynamics. I. Flows. Princeton: Princeton University Press 1967

11. Green, M.S.: J. Chem. Phys. 19, 1036 (1951); Lebowitz, J.L. : Phys. Rev. 114, 1192 (1959)

12. Vaserstein, L.N.: Commun. Math. Phys. 69, 31-56 (1979)

Communicated by A. Jaffe

Received July 29, 1980 